\title{
O modo Indicativo II em Tenetehára: considerações históricas
}

\author{
Tabita Fernandes da Silva ${ }^{1}$
}

\begin{abstract}
Resumo
O presente artigo trata do modo indicativo II nas duas línguas Tenetehára - o Tembé e o Guajajára - com enfoque nas mudanças atestadas desde os primeiros registros dessas línguas até o momento presente. A análise leva em consideração as descrições disponíveis sobre o tema em outras línguas da família Tupí-Guaraní, particularmente as descrições e análises do Tupinambá por Anchieta (1559), Figueira (1888) e Rodrigues (1953). O trabalho revela que o modo Indicativo II em Tembé e em Guajajára, embora preservado em várias situações, apresenta inovações em pleno desenvolvimento as quais diferenciam essas duas línguas das demais línguas da família Tupí-Guaraní em que a tendência tem sido o desaparecimento total desse modo como ocorreu em Ka'apór, em Zo’é e em Emerrilon.

Palavras-chave: Modo indicativo II. Tembé, Guajajára. Família Tupí-Guaraní. Mudança linguística.
\end{abstract}

\begin{abstract}
This paper deals with the II indicative mood in both languages Tenetehára - Tembé and Guajajára focusing on changes attested from the earliest records of these languages to the present moment. The analysis takes into account the descriptions available on the subject in other languages of the Tupí-Guaraní family, particularly descriptions and analyzes of Tupinambá by Anchieta (1559), Figueira (1888) and Rodrigues (1953). The paper reveals that the mode II indicative in Tembé and Guajajára, although preserved in various situation, in full development presents innovations witch differentiate these two languages from other languages of the Tupí-Guaraní family where the trend has been the way of total disappearance as occurred in Kaapór, in Zo’é and Emerrilon.
\end{abstract}

Keywords: II Indicative mode. Tembé. Guajajára. Tupí-Guarani family. Linguistic change.

\section{Introdução}

As descrições mais antigas sobre modos verbais na família Tupí-Guaraní são as de Anchieta (1559) e Figueira (1888) sobre o Tupinambá, e as de Montoya (1876) e Restivo (1893) sobre o Guaraní Antigo. Rodrigues (1953), baseado nesses autores, ao analisar a morfologia do verbo Tupinambá, mostra que esta língua fazia distinção entre dois grupos de formas verbais: as formas remáticas (as formas propriamente verbais) e as formas onomáticas (as de natureza nominal). As formas verbais de natureza remática identificadas no

1 Universidade Federal do Pará/ Campus Universitário de Bragança, tabita@ufpa.br. 
Tupinambá foram o Indicativo I (ou remático), o Permissivo, o Imperativo, o Gerúndio e o Nome relativo. As de natureza onomática eram o Indicativo II, (ou onomático), o Subjuntivo e nomes de ação, de agente, de circunstância, de objeto, de paciente, de agente habitual e de propensão (Rodrigues 1953:126).

\section{O Modo Indicativo I em Tembé e em Guajajára}

Rodrigues (1953:126) mostra que o modo indicativo I em Tupinambá exprimia a 'simples realização do processo verbal ('vi uma canoa'/ 'eu fui'). Exemplos do modo Indicativo I nas duas línguas Tenetehára são apresentados a seguir:

\section{Tembé}

Exemplos:

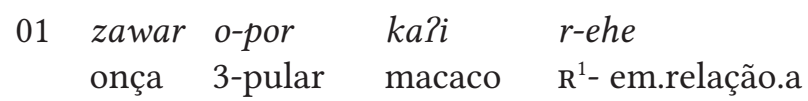

'a onça pulou no macaco'

02 a-ha kwej paragomin-pe

1-ir RLz Paragominas-LOC

'eu já fui a Paragominas'

03 a-ha ram paragomin-pe

1-ir PROJ1 Paragominas-LOC

'eu vou a Paragominas'

04 u-hem o-ho pihaw ete ahi

3-chegar 3-ir de manhã INT3 INT2

'ela vai chegar de manhãzinha'

05 kwei tua?u ?ai u-ririj Tetê aquele-vis velho ATN 3-tremer INT1 'aquele velhinho se tremia todo'

\section{Guajajára}

Exemplos:

06 awa u-tim manitok

homem 3-plantar mandioca

'o homem planta mandioca'

$\begin{array}{lllll}07 & \text { a?e } \quad \text { o-poj } & \text { u-emaw } & \text { zawar } & \text { ri?i } \\ \text { ele 3-alimentar } & \mathrm{R}^{4} \text {-animal de estimação } & \text { cachorro } & \text { AFIRM } \\ \text { 'ela alimentou o cachorro' } & & \end{array}$ 


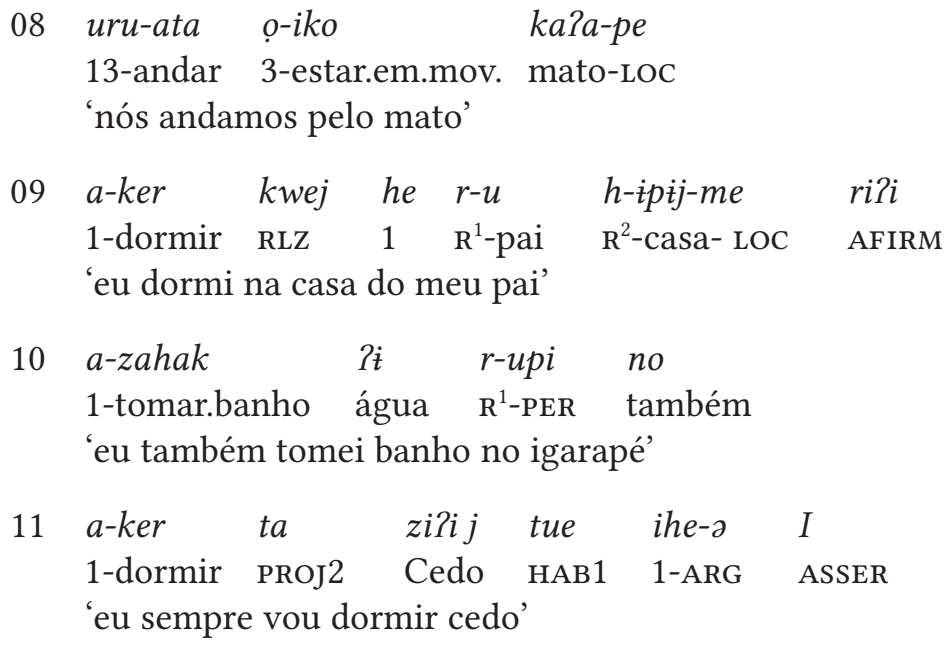

\section{O Modo Indicativo II em Tembé e em Guajajára}

De acordo com Rodrigues (1953), o modo Indicativo II era uma variedade de indicativo encontrada no Tupinambá, que ocorria quando uma circunstância antecedia um predicado com sujeito de primeira ou de terceira pessoa. $\mathrm{O}$ verbo, nesse contexto, não recebia prefixos pessoais, mas prefixos relacionais. Se o determinante do verbo formasse com este uma unidade sintática, o verbo recebia o prefixo $\mathrm{R}^{1}$; se, do contrário, o verbo recebia o prefixo $\mathrm{R}^{2}$. No verbo Tupinambá havia, ainda, o sufixo do modo Indicativo II, que tinha nessa língua dois alomorfes: o alomorfe $-\boldsymbol{i}$ que ocorria em temas terminados em consoantes e o alomorfe $-\boldsymbol{w}$ em temas terminados por vogais.

Em Tenetehára o modo Indicativo II ainda é expresso, tanto em Tembé quanto em Guajajára. Nas seções seguintes são apresentadas as expressões do modo Indicativo II em Tenetehára, considerando os dados do Tembé conforme os registros de Cyriaco Baptista (1932), Boudin (1966), e Carvalho (2001) e os do Guajajára por Bendor-Samuel (1972) e Harrison (1986).

\subsection{O Modo Indicativo II em Tembé desde os primeiros registros 2.1.1 O registro do Indicativo II em Tembé por Cyriaco Baptista (1932)}

Atualmente em Tembé o modo Indicativo II se realiza pela combinação de temas verbais com prefixos relacionais e com o sufixo desse modo - $\boldsymbol{n}$ (depois de vogal) e - $\varnothing$ (depois de consoante). Na coletânea de textos de Cyriaco Baptista $^{2}$ (1932) o modo Indicativo II ocorre com os verbos - ho 'ir' e - eko 'estar. em.movimento', ambos terminados em vogal e combinados, portanto, com o

2 Os fragmentos extraídos dos textos de Cyriaco Baptista foram, aqui, mantidos com a mesma escrita do texto original. 
alomorfe $-\boldsymbol{n}$ do sufixo desse modo. A seguir alguns exemplos desses verbos no modo Indicativo II.

Exemplos com os verbos - ho 'ir' e -eko -iko 'estar.em.movimento'

$-h o$ 'ir'

12 Iizêkuêhê koémirê; Ohóui zêkuêhê nó. Názáuê Zêkuêhê

'Disse em certo tempo ia indo em certo tempo de novo. Assim em certo tempo

Ihan Iko. Upuranupa Purunarỹ. Ohó

estava indo. Perguntando.' (Cyriaco Baptista 1932:389)

13 Umunãzauê Zêkuêhê aypó Omo Uariuaỹr Hekanynô.

Uma vez aquelle filho de estava de novo.

14 Upau Zêkuêhê

Acabou em certo tempo (Cyriaco Baptista 1932:388.)

\section{-eko -iko 'estar.em.movimento'}

15 Munã Zauê Zêkuêhê aypó 'Ahy' Hekan uzetanonga. Uair izeça'Uma vez em certo tempo estava a preguiça fazendo um encanto. Quando o filho

kukarirê Uzêupir zêkuêhê uicó. Apuihy Zêkuêhê ico Uzêupiâ appareceu Trepando em certo tempo elle estava. Disse em certo tempo dizendo e trepando' (Cyriaco Baptista 1932:386)

16 Umunazâuê Zêkuêhê aypó támánuá Hékan uzétanônganú Tucumãiu 'D'esta forma em certo tempo aquelle tamanduá estava em encanto.

Tucumanzeiro...' (Cyriaco Baptista 1932:380)

No exemplo 12 o tema - $h a$ combina-se com o relacional de não-contigüidade $\boldsymbol{i}$ - e com o alomorfe - $\boldsymbol{n}$ do modo Indicativo II. Essa forma é motivada pela presença do advérbio nazawê 'assim', 'dessa forma' que precede o predicado. No exemplo 16 o tema verbal -eko combina-se com o alomorfe $\boldsymbol{h}$ - do prefixo relacional de não- contigüidade, mas com o mesmo alomorfe $\boldsymbol{- n}$ do modo Indicativo II por ser também terminado por vogal.

\subsubsection{O registro do Indicativo II em Tembé por Boudin} (1966)

Nos registros de Boudin (1966), o sufixo do modo Indicativo II é seguindo consoante e - $\boldsymbol{n}$ variando com - $\boldsymbol{n} \boldsymbol{i}$ seguindo vogal. Exemplos do modo Indicativo II são encontrados em abundância. A presença de uma expressão 
de natureza adverbial nos dados de Boudin também é freqüente. A título de ilustração apresentamos exemplos com os verbos - ho "ir" e -ekó -ikó "estar. em.movimento" e com os verbos mano "morrer" e mume?ú "narrar", "contar", "relatar".

-ho 'ir'

$\begin{array}{lll} & \text { hupi-katu } & \text { i-ho- } \boldsymbol{n}(\boldsymbol{i}) \\ \text { 'está (vai) certo!' } & \text { 'está direito" (Boudin 1966:66) }\end{array}$

Boudin traduz a expressão hêkó-n(i) como 'estar', 'ficar', 'permanecer', 'perdurar', 'conservar-se'.

\subsubsection{O Modo Indicativo II em Guajajára segundo as análises de Bendor-Samuel (1972) e Harrison (1986)}

Bendor-Samuel (1972) não apresenta detalhamento sobre o modo Indicativo II em Guajajára. Denomina esse modo de "inverted sentence". Harrison, (1986:417), por sua vez, ao tratar de elementos oblíquos topicalizados em Guajajára, mostra que, se um advérbio é movido para o início da sentença ou para antes do verbo, sendo o sujeito de terceira pessoa, o verbo recebe a marca de (ergativo) absolutivo e o elemento oblíquo topicalizado é registrado no verbo por meio do sufixo $-\boldsymbol{n}$ (após vogal) e $\sim-\varnothing$ (após consoante). Esta situação descrita por Harrison corresponde ao que Rodrigues (1953) chama de Indicativo II.

A seguir exemplos de Harrison (1986: 418) os quais mostram o contraste entre sentenças com o elemento oblíquo topicalizado e outras com o oblíquo em posição não topicalizada em Guajajára.

18. w-iko a?e-pe

3-be there-at

'He is there' (Harrison 1986:418)

19 a?e-pe Ø-heko-n

there-at 3SG-be-OBL.TOP

'He is there' (Harrison 1986:418)

20 ka?a-pe ure-reraha-n

jungle-to 1PL.EXCtake-OBL-TOP

'(He) took us to the jungle' (Harrison 1986:418)

Segundo Harrison, esse tipo de construção já estava caindo em desuso em alguns dialetos do Guajajára, ocorrendo com verbos intransitivos tais como 'ir', 'vir', 'ser/estar' e outros destes derivados. 


\subsection{O Modo Indicativo II em dados mais recentes do Tembé e do}

\section{Guajajára}

Os estudos de Carvalho (2001) sobre o Tembé mostram que o modo Indicativo II, embora ainda ativo, à época de sua pesquisa, já tinha uso restrito e com algumas particularidades: sua ocorrência estava restrita a situações em que o sujeito era de terceira pessoa, sendo acionado apenas quando o predicado fosse afirmativo. Carvalho mostra que as estratégias de negação usadas no modo Indicativo II eram as mesmas usadas no modo indicativo I, mas também havia casos em que a negação era feita por meio da partícula ni?í e que dois grupos de falantes já estavam usando àquela altura, o modo Indicativo II de forma não sistemática.

\subsubsection{O sufixo $-n \sim-\varnothing$ na expressão do Modo Indicativo II em Tembé e em Guajajára}

Em nossos dados (Silva 2010), confirma-se o já descrito em estudos anteriores sobre o modo Indicativo II no Tembé e no Guajajára. Mas, como mostramos adiante, o sufixo do modo Indicativo II encontra-se em plena expansão para outros contextos morfossintáticos não mais relacionado à anteposição de uma circunstância ao predicado. Nesta seção apresentamos dados de nossa pesquisa e traçamos discussões adicionais a respeito da manifestação atual desse modo nas duas línguas Tenetehára. A seguir apresentamos exemplos do Modo Indicativo II em diferentes contextos de ocorrência e as inovações que essas línguas têm apresentado para a expressão desse modo.

\section{Tembé}

\section{Com o verbo - eko 'estar em movimento'}

Exemplos:

lá $\quad \mathrm{R}^{2}$-estar.em.mov.-INDII Paragominas

'ela está lá em Paragominas'

22 zapukaj r-a?ir h-ake h-eko-ni

galinha $\mathrm{R}^{1}$-filhote $\mathrm{R}^{2}$-perto $\mathrm{R}^{2}$-estar.em.mov.- INDII

'aquele pintinho está perto dela'

Com o verbo - ho 'ir'

23 kwehe i-ho-n Kwej

PASS.REM. $\quad \mathrm{R}^{2}$-ir- INDII RLZ

'ele foi embora desde muito tempo' 


\section{4 kuda ywer uaj iru ramo i-ho-ni \\ mulher COL PL ASS $\mathrm{R}^{2}$ - ir- INDII \\ 'foi na companhia das mulheres que ela foi'}

\section{Em sentenças interrogativas e negativas}

No caso das sentenças interrogativas abaixo é o caso translativo ramo que aciona o modo indicativo II.

\begin{tabular}{|c|c|c|c|c|}
\hline $\begin{array}{l}\text { amo } \\
\text { quem }\end{array}$ & $\begin{array}{l}\text { r-aikwer } \\
\mathrm{R}^{1} \text {-atrás }\end{array}$ & $\begin{array}{l}\text { ramo } \\
\text { TRANS }\end{array}$ & $\begin{array}{l}\text { kwaharer } \\
\text { menino }\end{array}$ & $\begin{array}{l}h \text {-eko- } \boldsymbol{n} \\
\mathrm{R}^{2} \text {-estar.em.mov.-INDII }\end{array}$ \\
\hline
\end{tabular}

Note-se que, no exemplo seguinte, o morfema do caso translativo tem um valor associativo.

$\begin{array}{lllll}26 & n & \text { akwaw amo } & \text { iru-ramo } & \text { i-ho-ni } \\ \text { NEG saber quem companheiro-TRANS } & \mathrm{R}^{2} \text {-ir-INDII } \\ \text { 'eu não sei com quem ele vai' } & \end{array}$

Nos exemplos a seguir era esperado que os temas verbais ocorressem no modo indicativo II, pois são precedidos por uma construção com a partícula mehe, mas esse modo não é acionado:

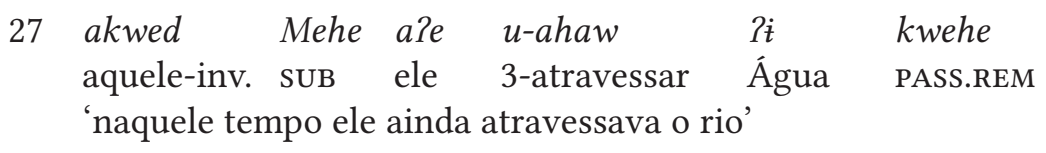
28 akwed mehe ale u-pir we ?i-wi aquele-inv. SUB ele 3-carregar CONT água- DAT 'naquele tempo ele ainda carregava água do rio'

\section{Guajajára}

\section{Com o verbo - eko 'estar em movimento'}

Sem a circunstância antecipada, nota-se que o modo indicativo II não é acionado, como nos exemplos 29 e 33 a seguir, diferente dos demais exemplos em que este é acionado, um tipo de construção ainda ativo no Guajajára.

$$
\begin{array}{llllll}
29 & \text { he } & \varnothing-h i & u \text {-iko } & \text { ko-pe } & \text { kuri } \\
& 1 & \mathrm{R}^{1} \text {-mãe } & \text { 3-estar.em.mov. } & \text { roça-Loc } & \text { agora } \\
\text { 'minha mãe está na roça agora' } & &
\end{array}
$$

$$
\begin{aligned}
& 30 \text { ko-pe he Ø-hit h-eko-n } \\
& \text { roça-LOC } 1 \quad \mathrm{R}^{1} \text {-mãe } \quad \mathrm{R}^{2} \text {-estar.em.mov.-INDII } \\
& \text { 'na roça a minha mãe está' }
\end{aligned}
$$


31 ko-pe h-eko-n a?e roça- LOC $\mathrm{R}^{2}$-estar.em.mov.-INDII ele 'na roça ele está'

32 t-ipuz-pe h-eko-n a?e wo $\mathrm{R}^{4}$-casa- LOC $\mathrm{R}^{2}$-estar.em.mov.-INDII ele $\mathrm{PL}$ 'na casa eles estão'

Com o verbo - ho 'ir'

33 he Ø-hi o-ho kwez ko-pe

$1 \quad \mathrm{R}^{1}$-mãe 3-ir RLZ roça-LOC 'minha mãe foi pra roça'

34 ko-pe he Ø-hit i-ho-n kwez roça- LOC $1 \quad \mathrm{R}^{1}$-mãe $\mathrm{R}^{2}$-ir-INDII RLZ 'pra roça minha mãe foi'

35 ko-pe i-ho-n kwej roça- LOC $\mathrm{R}^{2}$ - ir-INDII RLZ 'pra roça ele foi'

36 irikaw-pe i-ho-n kwej igarapé-LOC $\mathrm{R}^{2}$ - ir-INDIIRLZ 'pro igarapé ele foi'

\subsubsection{O sufixo - $i$ na expressão do Modo Indicativo II em}

\section{Tembé e em Guajajára}

Embora o modo Indicativo II em Tembé e em Guajajára seja expresso pelo sufixo $-\boldsymbol{n} \sim-\emptyset$, há casos em que certos verbos terminados em consoante recebem o sufixo - $\boldsymbol{i}$ sempre que a circunstância é antecipada ao verbo. Exemplos com e sem esse sufixo são apresentados a seguir:

\section{Tembé}

\section{Com verbo intransitivo:}

$$
\text { -ur'vir' }
$$

37 pihawe Ø-tur-i ram aipo nehe amanhã 3-vir- INDII PROJ1 INF INTEN 'com certeza o rapaz vem amanhã'

No exemplo que segue, embora o verbo venha com o sufixo - $\boldsymbol{i}$, combina-se com prefixos subjetivos, o que é uma inovação marcante. 
Raw apiaw mujte u-itur-i

este-deit/em.pé homem longe 3-vir-INDII

'este homem veio de muito longe'(em pé)

Já no exemplo seguinte, a forma verbal marcada por - $\boldsymbol{i}$ parece funcionar mais como auxiliar no modo gerúndio:

39 ne r-iwir u-hem Ø-ur-i

$2 \quad \mathrm{R}^{1}$-irmão 3-vir 3-vir-INDII

'foi seu irmão quem chegou'

Os dados seguintes mostram claramente formas verbais de predicados principais, precedidos por expressão adverbial, flexionados pelo morfema $-\boldsymbol{i}$ do indicativo II, mas também flexionados por prefixos pessoais e não por prefixos relacionais.

-Pəm 'estar.em.pé'

40 kamut $i$ r-uadar ramo mukaw u-?əm-i

pote $\quad \mathrm{R}^{1}$-lado TRANS espingarda 3-estar.em.pé- INDII

'é ao lado do pote que a espingarda está'

41 he r-uadar ramo mukaw u-?am-i

$1 \quad \mathrm{R}^{1}$-lado TRANS espingarda 3-estar.em.pé- INDII

'é do meu lado que a espingarda está'

\section{-in 'estar sentado'}

42 i⿱ r-eke he r-ipij u-in-i

água $\quad \mathrm{R}^{1}$-perto $\quad 1 \quad \mathrm{R}^{1}$-casa $\quad 3$-estar.sentado-INDII

'é perto do rio que fica a minha casa'

43 kamut $\int i$ r-eke dapepo u-in-i

pote $\mathrm{R}^{1}$-perto panela 3-estar.sentado-INDII

'a panela está perto do pote'

-Par 'cair'

44 kwaharer ?ar ramo ita $u$-?ar-i

menino em cima pedra 3-cair-INDII

'foi em cima do menino que a pedra caiu'

\section{Guajajára}

Nota-se que, nos exemplo 45 e 46 do Guajajára, sem a circunstância antecipada, o indicativo II não é acionado. No exemplo 47, no entanto, com a circunstância precedendo o núcleo do predicado, o sufixo - $\boldsymbol{i}$ foi usado para marcar o modo indicativo II. 
$-u r^{\prime}$ vir'

45 u-zur kwez amon upaw ire

3-vir RLZ chuva CES depois

'ele chegou depois da chuva'

46 Ø-ur putar ru?u Francisco se pihawe

3-vir PROJ2 DUB Francisco aqui Amanhã

'será que Francisco vem aqui amanhã?'

47 aman upaw Ire u-zur-i Kwez

chuva CES depois 3-vir-INDII RLZ

'depois da chuva ele chegou'

Outro traço inovador nas duas línguas Tenetehára é a extensão da ocorrência do sufixo - $\boldsymbol{i}$ do modo indicativo II com formas verbais núcleos de predicado com sujeitos de $1^{\mathrm{a}}, 2^{\mathrm{a}}$ e $3^{\mathrm{a}}$ pessoas.

\section{Tembé}

Exemplos:

-deruzar -zerudar 'acreditar', 'confiar'

48 amo r-ehe ere-derudar-i

quem $\quad \mathrm{R}^{1}$-em.relação.a 2-confiar-INDII

'você confia em quem?'

\section{Guajajára}

Exemplos:

49 karu mehe ere-zur-i

ontem 2-vir-INDII

'ontem você chegou'

\section{-ho 'ir'}

50 maj?u paw Ire uru-momor ta Bol o-ho-j

comer CES depois 13-jogar PROJ2 Bola 13-ir- INDII

'depois do almoço nós vamos jogar bola'

51 amən ipik ire a-mareko tatá a-ha-j

chuva fim depois 1-trabalhar PROJ2 1-ir- INDII

'depois da chuva eu vou trabalhar'

O exemplo seguinte ilustra a ocorrência do sufixo $-\boldsymbol{i}$ do modo indicativo coocorrendo com um prefixo pessoal de $3^{\mathrm{a}}$ pessoa em Tembé. 


\section{-mudar -muzar 'enrolar'}

$\begin{array}{llll}52 \text { akwed mehe a?e } & \text { u-mudar-i } & \text { pitim-a } \\ \text { aquele-inv. tempo ele } & \text { 3-enrolar-INDII } & \text { fumo-ARG } \\ \text { 'naquele tempo ele ainda enrolava o fumo' } & \end{array}$

Os exemplos com o sufixo - $\boldsymbol{i}$ mostram uma grande mudança na língua. Por um lado, há vestígios do antigo modo Indicativo II em que a forma - $\boldsymbol{n}$ alterna com a forma - ni depois de tema terminado em vogal e - $\varnothing$ depois de consoante. Por outro lado, verifica-se uma extensão do uso do sufixo do modo indicativo II para temas flexionados por prefixos pessoais quando núcleos de predicados precedidos por expressões adverbiais. Essa extensão parece, à primeira vista, uma inovação relativa ao modo Indicativo II. Por contrariar restrições ativas em um estágio anterior da língua, trata-se de uma variante. Há, ainda, situações em que um tema verbal que se combina com o sufixo - $\boldsymbol{i}$ não é núcleo do primeiro predicado que segue a expressão adverbial, mas o último predicado da sentença que funciona como predicado auxiliar conforme mostram os seguintes exemplos:

\section{Guajajára}

Exemplos:

53 amən ipik ire a-zur putar nehe
chuva fim depois 1-vir PROJ2 INTEN
'depois da chuva eu venho'

\begin{tabular}{|c|c|c|c|c|c|c|}
\hline $\begin{array}{l}\text { maj?u } \\
\text { comer }\end{array}$ & $\begin{array}{l}\text { paw } \\
\text { CES }\end{array}$ & $\begin{array}{l}\text { Ire } \\
\text { depois }\end{array}$ & $\begin{array}{l}\text { uru-momor } \\
\text { 13-jogar }\end{array}$ & $\begin{array}{l}\text { ta } \\
\text { PROJ2 }\end{array}$ & $\begin{array}{l}\text { Bol } \\
\text { Bola }\end{array}$ & $\begin{array}{l}o-h o-j \\
13 \text {-ir- INDII }\end{array}$ \\
\hline
\end{tabular}

Finalmente um fato muito inovador é a ocorrência do modo Indicativo II em situações em que o predicado é precedido por qualquer elemento topicalizado e não exclusivamente por uma expressão adverbial como o descrito para as línguas conservadoras da família Tupí-Guaraní.

\section{Tembé}

Exemplos:
55 a?e kwaharer u-madan-i
ele menino 3-empurrar-INDII
'foi ele que empurrou o menino'
56 ne Kwaharer ere-madan-i
2 Menino 2-empurrar-INDII
'foi você que empurrou o menino'


57 manitok a?e u-tim-i

mandioca Ele 3-plantar-INDII

'era mandioca o que ela plantava'

$58 \quad 2 \dot{i} \quad u-e n u-j \quad$ wo

água 3-pedir-INDII 3.PL

'é água o que eles estão pedindo'

\section{Guajajára}

Exemplos:

Assim como ocorre em Tembé, em Guajajára também verifica-se a ocorrência ora do sufixo - $\boldsymbol{n}$, ora do sufixo - $\boldsymbol{i}$ precedidos por expressão adverbial. Os temas verbais recebem tanto os prefixos pessoais quanto os relacionais.

\section{Com o verbo - ho 'ir'}

59 aman ipik Ire uru-momor Ta

chuva fim depois 13-jogar PRoj2

bol o-ho-j ure-əj

bola 3-ir-INDII 13-ARG

'depois da chuva nós vamos jogar'

Há, também, casos em Guajajára em que, mesmo havendo as condições ideais para o uso do modo Indicativo II, este não se realiza, como nos exemplos a seguir:

Com a circunstancia antecipada:

-eko 'estar em movimento'

60 ko-pe u-ata $\varnothing$-iko

roça- LOC 3-andar 3corr-estar.em.mov.

'pra roça ele foi'

\section{$-h o$ 'ir'}

61 pitun ete a?i mehe a-hem a-há Kwez noite INT3 ATN SUB 1-sair 1-ir RLZ 'à noitinha eu saí'

62 pihawe ete ahi aha putar Ihe manhã INT3 INT 2 1-ir $\operatorname{PROJ} 21$ 'de manhã eu vou'

63 zilij zahi za-ha ta ahi Nehe cedo manhã 12-ir PROJ2 INT2 INTEN 'nós vamos de manhã' 
64 pihaw o-ho putar a?e

noite 3-ir PROJ2 Ele

'de noite ele vai'

65 aman ipik ire a-ha putar Ihe

chuva fim depois 1-ir PROJ2 1

'depois da chuva eu vou'

Há situações, no entanto, em que o modo Indicativo II é usado mesmo sem circunstância alguma claramente expressa na sentença.

$\begin{array}{lll}66 & \text { i-ho-n } & \text { Kwej } \\ & \mathrm{R}^{2} \text {-ir- INDII } & \text { RLZ } \\ & \text { 'ele foi' } & \end{array}$

Os dados apresentados até aqui evidenciam que o modo Indicativo II em Tembé e em Guajajára, embora preservado em várias situações, apresenta inovações em pleno desenvolvimento as quais diferenciam essas duas línguas das demais línguas da família Tupí-Guaraní em que a tendência tem sido o desaparecimento total desse modo como ocorreu em Ka'apór, em Zo'é e em Emérillon.

\subsection{Considerações sobre o Modo Indicativo II em Tenetehára}

Os dados discutidos até aqui mostram que as duas línguas Tenetehára têm mudado em direções análogas em vários aspectos, mas o Guajajára tem sido, ao mesmo tempo, mais conservador e também mais inovador quanto ao modo indicativo II. Na situação atual do Guajajára há uma variação entre formas no indicativo II com sufixo de modo e relacionais e formas com sufixo de modo e prefixos pessoais. Essa situação leva ao entendimento de que os prefixos subjetivos do Tenetehára na variante Guajajára não são mais exclusivos do indicativo I, mas das duas variedades de indicativo, I e II. Os dados mostram que as formas combinadas com esses prefixos tornam-se produtivas enquanto que as formas com relacionais têm seu uso reduzido tanto em Tembé quanto em Guajajára. Observa-se, também, que, na língua Tembé, o Indicativo II passa a ser usado como forma condicionada a uma topicalização, independentemente da natureza do elemento topicalizado.

Finalmente, no que diz respeito às formas fonológicas do morfema do indicativo II em Guajajára e em Tembé, há evidências de que compartilham similaridades com o Guajá e com o Guaraní Antigo. Ora, sendo o Guajá uma língua do sub-ramo VIII, há a possibilidade de que a forma - $\boldsymbol{n i}$ do indicativo II nessa língua tenha sido um desenvolvimento do contato dos seus falantes com falantes do Tenetehára. 
Há também indicações de que o Indicativo II do Tenetehára tenha sido restrito a construções com sujeito de terceira pessoa, mas que o novo modo indicativo II em desenvolvimento tenha se estendido para todas as pessoas.

De toda forma, a presença de um alomorfe - $\boldsymbol{n} \boldsymbol{i}$ do modo indicativo II em Tenetehára faz dele uma das línguas mais conservadoras do ramo setentrional, quanto a essa forma, visto que a mesma só é encontrada no Guaraní Antigo, além do Guajá.

\section{Referências}

Bendor-Samuel, David. 1972. Hierarchical structures in Guajajara. Summer Institute of Linguistics Publications in Linguistics and Related Fields, 37. Norman: Summer Institute of Linguistics. XIV.

Boudin, M. H. 1966. Dicionário de Tupi-Moderno. São Paulo: Faculdade de Filosofia, Ciências e Letras de Presidente Prudente.

Carvalho, M.G.P. 2001. Sinais de Morte ou de Vitalidade? Mudanças estruturais na Língua Tembé. Dissertação (Mestrado em Linguística) UFPA.

Figueira, Padre Luiz. 1880. Arte de Grammatica da Lingua Brasilica. Typographia e Litographia a vapor de Lombaerts \& C. Ourives n. 7. Rio de Janeiro.

Harrison, Carl H. 1986. Verb Proeminence, Verb Initialness, Ergativity and Typological Disharmony in Guajajára. In: Handbook of Amazonian Languages - volume I. Desmond Derbchire C. Pullum, Geofrey K. ed. Berlim; New York; Amsterdam: Mouton de Gruyter.

Rodrigues, A. D. 1986. Línguas brasileiras: para o conhecimento das línguas indígenas. São Paulo: Loyola. . 1985. A classificação do tronco lingüístico Tupi. Revista de Antropologia 12(12):99-104.

. 1996. Argumento e predicado em Tupinambá. Boletim da Associação Brasileira de Lingüística 19:57-68. Maceió.

Rodrigues A. D.; A. S. A.C. Cabral. 2001. Revendo a classificação interna da família Tupi-Guaraní. In: Actas do I Encontro Internacional do GTLI da ANPOLL. Belém: EDUFPA.

Snethlage, Emil Heinrich. 1932. Worte und texte der Tembé-indianer aufgezeichnet von Cyriaco Baptista (Tembé). In De La Revista Del Instituto de Etnologia, Tomo II, pp. 347-393. Tucumán.

Data recebimento: 05/01/2013

Data aceite: 15/08/2013 